Cinémas

Revue d'études cinématographiques

Journal of Film Studies

\title{
BOYER, Martine, L'Écran de l'amour. Cinéma, érotisme et pornographie, 1960-1980, Paris : Plon, 1990, 185 p.
}

\section{Ratiba Hadj Moussa}

Volume 2, numéro 2-3, printemps 1992

URI : https://id.erudit.org/iderudit/1001087ar

DOI : https://doi.org/10.7202/1001087ar

Aller au sommaire du numéro

Éditeur(s)

Cinémas

ISSN

1181-6945 (imprimé)

1705-6500 (numérique)

Découvrir la revue

Citer ce compte rendu

Moussa, R. H. (1992). Compte rendu de [BOYER, Martine, L'Écran de l'amour.

Cinéma, érotisme et pornographie, 1960-1980, Paris : Plon, 1990, 185 p.]

Cinémas, 2(2-3), 231-235. https://doi.org/10.7202/1001087ar d'utilisation que vous pouvez consulter en ligne.

https://apropos.erudit.org/fr/usagers/politique-dutilisation/ 
BOYER, Martine, L'Écran de l'amour. Cinéma, érotisme et pornographie, 1960-1980, Paris: Plon, 1990, 185 p.

Ce livre est un essai historique sur le cinéma érotique et pornographique (1960-1980). Sans tenter de marginaliser l'apport du cinéma en matière de révolution sexuelle et de moeurs, Boyer considère que les grands mouvements sociaux étaient beaucoup plus intéressés par «le contrôle du champ imaginaire» que par celui du champ cinématographique.

La libération de ce champ a permis au cinéma d'interroger le niveau latent qui l'anime, à savoir la représentation du sexe et de la sexualité, et de former un genre complètement voué à cette dernière. Cependant, entre le cinéma à grand public et le cinéma pornographique, il existe plus d'un lien. Selon Boyer, les films importants, «de prestige», ont parties liées avec les films porno dans le mesure où ils renvoient implicitement et explicitement à ces dernières. Les films porno sont alors le niveau refoulé des films de prestige. Ce qui justifie la force d'un Godard par exemple, c'est la lucidité qu'il a montré vis-à-vis de cette vérité: «Il [Godard] n'a pas en fait cessé d'affirmer le cinéma comme art de l'obscénité et donc le porno comme $L E$ sujet — refoulé — du cinéma» (p. 49). Si le porno a obligé l'Autre cinéma à «se mettre a nu» en exposant ses vedettes, alors réticentes à toute dénudation, celui-ci en retour a fortement participé a la transformation de son mode de raconter. C'est le cas de la Nouvelle Vague par exemple, qui a indirectement influencé les sexy en délaissant l'intrigue au profit de l'évolution des personnages, ou en introduisant des séquences qui ne la justifient pas. D'où «l'avènement du sexe à l'écran comme sujet de film à part entière, pour ne pas dire comme $L E$ film sous-jacent de tout film» (p.144).

L'hypothèse de la dépendance interfilmique ne semble pas être affectée par les «spécialités nationales» dont on trouve une classification assez tranchée dans le livre:

1. Les naturistes qui proviennent, comme on s'en doute, des pays scandinaves, de Suisse et de Grande-Bretagne. Ces films inaugurent la décennie 1960. La représentation du nu, féminin et masculin, donnait à voir une conception du corps comme un «en 
soi» et relevait d'une conception d'une nudité «aboutie» qui fait fi des rituels fétichistes. Cette représentation des corps en symbiose avec leur milieu naturel, révélant un souci évident de l'écologie, n'était ni explosive ni menaçante parce qu'elle tablait sur «le corps multiple» et non sur le corps singulier qui constitue la représentation de la marge même.

2. Les hard-cores américains dont le chantre fut Russ Meyer qui, si on en juge par ses propos (en annexe), sont loin d'être aussi distants de leur culture d'origine, de «L'Amérique profonde». C'est pour stigmatiser celle-ci que les hard-cores ont versé dans la vision grandiloquente du cadre de vie, un peu comme le font les séries télévisuelles Dallas et Dynasty.

3. Les sexy music-hall italiens qui réfèrent carrément aux débuts du cinéma, à sa tradition du spectacle de foire. Ces films sont essentiellement composés de scènes de cabaret et de caféthéâtre. Ils consacrent le sexe comme objet d'exhibition. Après les films, «l'envol, écrit Boyer, sera irréversible». La particularité de ces films, qui ne mettent pas l'accent sur «le nu spirituel», est qu'ils «professionnalisent la nudité» et lui apposent le label de l'art. Ils déplacent ainsi de manière notable la conception naturalisant la nudité: «(...) elle n'est [désormais] plus qu'un relais du centre d'intérêt de l'oeil, et déjà un paradoxe trop embourbé de fétichisme» (p. 58).

4. Les films des docteurs allemands ont abordé frontalement la sexualité. Ce sont les médecins eux-mêmes qui, sous couvert de la science, ont produit ces films. Appuyés par des «scénarios médicaux» (visites chez le médecin), sertis par une fonction documentaire, balise à toute dérive, ces films débloquent la problématique du caché-montré. «La médecine peut aller droit au but, prendre en charge la sexualité pour la transformer en un culte de santé. Aux antipodes de l'érotisme "à franfreluches" dont raffolent les latins, le corps germanique se meut dans l'éclatante franchise de la chaîne généalogique» (p. 64). La préoccupation de ces films est de protéger et de préserver la nation allemande contre les perversions. Ils agissent comme une sorte de réglage étatique de la sexualité.

Après cette brève classification qui, comme on le voit, fait ressortir les caractères sociologiques de chaque pays, le reste du 
livre approfondit la question des films porno dans le contexte français. L'année 1975 a sonné le glas de ces films désormais classés dans la catégorie «X». Après une percée assez fulgurante au début des années 1970, les «X» connurent de plus en plus de difficultés. La loi les concernant les marginalisa; et la confusion entre le hard et le soft, le porno et l'érotique ne tarda pas à suivre: un «ordre sexuel s'installe avec sa dimension didactique, quasi pédagogique» (p. 101). L'effet censure n'est pas sans coïncidence avec les «problèmes rattachés au sexe». En effet, la vague politique de 1968 qui contesta le contrôle politique des corps et de la sexualité s'estompe au milieu des années 1970 pour donner naissance aux discours "psy», qui revalorisent «les problèmes sexuels» et produisent progressivement une équation dans laquelle chaque sujet-individu se trouve confronté solitairement à son problème. Déflagration tout à fait postmoderniste ou passage obligé d'un ordre de croyance à un autre? Pendant que la parole s'épanche sur les divans, la rigueur s'installe. Elle commence par les têtes, passe par les corps et s'arrête aux images.

Au plan social, cette rigueur s'accompagne par un croisement entre les lois de la censure (lois X) et l'année internationale de la femme, laquelle est abrogée par une série de lois, notamment celles qui autorisent l'interruption volontaire de la grossesse et la commercialisation de la pilule contraceptive. Mais si cette réalité permet une certaine théâtralisation du sexe, elle ne conduit pas à une mise en cause d'un imaginaire tout ordonné et déjà là (à preuve, l'officialisation du mouvement féministe sous forme de Secrétariat d'État et autres instances gouvernementales).

Au plan économique, le cinéma porno perd la suprématie qu'il détenait au début des années 1960. Il devient un chaînon de l'industrie du sexe, secondé par les vidéo-clubs, les bars, les sex shops, etc. Tous ces lieux servent de mise en cadre à la sexualité. Il s'agit d'une véritable «assomption de la chair à l'écran», assomption d'autant plus marquée que l'industrie qui distille la chair s'auto-reproduit et s'auto-suffit. Ce qui explique, aux Etats-Unis par exemple, la présence paradoxale et simultanée du fonctionnement en continu d'un sex-service et de l'existence de ligues prônant violemment le puritanisme. C'est la chair qui intéresse le porno et non le corps: «dans le porno, note l'auteure, le sexe et 
l'amour parlent à peu de choses près, le même langage. Ils mettent entre parenthèses l'érotique et son encombrante philosophie du corps» (p. 119). Cet aspect est, entre autres, l'effet de déréalisation propre au langage cinématographique; déréalisation qui est renforcée, dans le cas du porno, par une utilisation particulière du son. L'usage de la «musicomètre» en est un. Il consiste à enlever au son ses attributs trop anthropologiques (trop vivants) qui pourraient créer un déphasage et mettre en danger le statut de la fiction. Le passage de la vidéo change les données; il ne tente pas d'amortir le «trop réel» des scènes pornographiques, il les inscrit entièrement dans le «purement porno» (le son devient synchrone). L'entrée du porno dans l'espace privé bouleverse les données de cette époque. La fabrication des bandes se fait «sur mesure» et la version hard, parce que considérée comme plus réelle, prend de plus en plus de place. En parallèle à ce déplacement de la sphère publique à la sphère privée, les films érotiques se démarquent nettement des porno et s'installent comme genre, à part entière. Outre le fait que ce genre a «fait comprendre au cinéma que l'image détient le pouvoir de donner une impression de richesse à partir du contenu le plus misérable» (p. 134), il ouvre la voie aux films discodanse des annees 1980 et au phénomène des cultes du corps (culturisme, body building, etc.). On assiste à l'émergence d'un nouveau rapport au corps, à un rapport à soi basé sur le paraître. La douceur veloutée de la peau dans les films érotiques, l'assomption de la chair à l'écran font surface dans les «love stories» des films commerciaux. Le sexe devient parfois le sujet essentiel de nombreux films de grands réalisateurs (Godard, Fellini, Pasolini), et est infléchi fortement par la variable «psy». Dans ces films, on circule facilement dans les profondeurs de l'œdipe, on triture le désir et on cherche le besoin.

Que dire finalement du cinéma et de l'érotisme de l'après 1980 ? M. Boyer y voit une édulcoration de la représentation du sexe à l'écran. Les porno ne correspondent plus à aucune réalité; quant aux érotiques, ils se moulent tellement aux idées et images reçues et acceptées qu'ils font bon ménage avec la T.V. Ils ne font plus peur et prônent une philosophie de la douceur. Restent les grandes fictions qui misent tort sur la passion (cf. Diva de Beineix) qui comme on le sait, se vit beaucoup dans la solitude, ou alors elles 
se penchent sur la ménagerie - les ours, les gorilles, les dauphins.

Ce livre, à écriture franchement métaphorique (journalistique?) contient de nombreuses informations sur les genres porno et érotique. Néanmoins, il laisserait insatisfait-e-s ceux et celles qui préfèrent une démonstration et une argumentation plus approfondies: plusieurs hypothèses restent en plan et ne sont pas conduites à leur terme. Enfin, c'est un livre qui, même s'il réfère à plusieurs cinémas nationaux (cf. la classification proposée), reste enclavé dans l'hexagone et réduit ainsi la logique qu'aurait pu offrir une réflexion d'ensemble sur ce genre cinématographique.

À noter, en annexe, une bibliographie assez exhaustive, des entrevues avec des réalisateurs et une filmographie.

Ratiba Hadj Moussa

Université Laval 